$\mathrm{IC} / 97 / 207$

hep-ph/yyyyyyy

\title{
Signatures of Non-Universal Soft Breaking Sfermion Masses at Hadron Colliders
}

\author{
Amitava Datta 7 and Aseshkrishna Datta 7 \\ Department of Physics, Jadavpur University \\ Calcutta- 700 032, India, \\ and \\ M.K. Parida 国四 \\ International Centre for Theoretical Physics, Trieste, Italy.
}

\begin{abstract}
We identify several mass patterns, within the framework of $N=1 S U G R A$ with nonuniversal soft breaking masses for the sfermions, which may significantly alter $S U S Y$ signals and the current squark-gluino mass limits from the Tevatron. These effects are illustrated in a $S O(10) S U S Y$ GUT with an intermediate mass scale, but the conclusions are also valid in $S U S Y S O(10)$ models with grand deserts.
\end{abstract}

\footnotetext{
${ }^{1}$ E-mail: adatta@juphys.ernet.in

${ }^{2}$ E-mail: asesh@juphys.ernet.in

${ }^{3}$ E-mail:mparida@nehus.ren.nic.in

${ }^{4}$ Permanent Address:Physics Department,North-Eastern Hill University,Shillong 793022,India

${ }^{5}$ ICTP Associate
} 
A strong prediction of $N=1$ supergravity ( $S U G R A$ ) models with supersymmetry ( $S U S Y$ ) breaking due to the hidden sector is that the soft breaking parameters are universal [1]. The economy in the number of parameters and the resulting predictive power have made this model (referred to hereafter as the conventional scenario) particularly popular. Even the current experimental limits on the sparticle masses are often derived by assuming that their spectrum is indeed given by the conventional scenario.

It has, however, been emphasised in the literature for quite some time that nonuniversal soft $S U S Y$ breaking terms at some high scale may arise quite naturally within the SUGRA framework [2]. For example, even if strict universality holds at the Planck scale, non-universal soft breaking terms may arise at a lower scale due to renormalization effects. Within the frame work of a grand unified theory (GUT) such effects are calculable. One simply has to consider the evolution from the Planck scale to the GUT scale $\left(M_{G}\right)$ by using the Renormalisation Group (RG) equations of the underlying GUT [3]. It is, therefore, extremely important to study the impact of this nonuniversality on collider signatures of sparticle production.

Once we accept the possibility of non-universality of the soft breaking parameters of the full theory including heavy fields, through the above or any other suitable mechanism, an interesting consequence follows whenever the rank of the GUT group and/or some intermediate symmetry is reduced by spontaneous symmetry breaking [4, 5]. One obtains $D$-term contributions, which apriori can be quite significant in magnitude, leading to non-universal squark and slepton masses at a scale much higher than the electroweak $(\mathrm{EW})$ scale. As a result the low energy sparticle spectrum can be significantly different from that in the conventional scenario.

Some phenomenological consequences of these D-terms in the context of $S U S Y$ GUTs with grand deserts as well as models with intermediate mass scales have been considered in the literature [5]. In this letter, we focus on those aspects of the sparticle spectrum which may lead to collider signatures significantly distinct from the conventional ones and, hence, to revisions of the current mass limits of the sparticles.

For the purpose of illustration we have carried out our calculations in a $S O(10)$ 
SUSY GUT model with an intermediate mass scale $\left(M_{I}\right)$ [6] which utilises the particle spectrum predicted from superstring compactification[7]. It should, however, be emphasised that our conclusions are not crucially dependent on the intermediate scale. As we shall discuss below, many of our conclusions hold even if the GUT group breaks down to the standard model $(S M)$ gauge group through a single step.

The model with an intermediate scale that we have considered is highly attractive for various aesthetic and phenomenological reasons. It provides a natural explanation for small neutrino masses through the see-saw mechanism [8], which has been shown to operate in string-inspired $S O(10)$ in the absence of the higher dimensional representation 126 [9]. With $M_{I} \simeq 10^{12}-10^{13} \mathrm{GeV}$, the predicted neutrino mass spectrum allows $\nu_{\tau}$ as a prospective candidate for hot dark matter of the universe and an explanation of the solar neutrino puzzle through $\nu_{e}-\nu_{\mu}$ oscillation in a manner similar to that discussed at length for intermediate scale models [10] via MSW mechanism 111.

Among the various candidates for the intermediate gauge group the Pati - Salam group $G_{224}=S U(2)_{L} \otimes S U(2)_{R} \otimes S U(4)_{c}$ with $g_{2 L}=g_{2 R}$ 12 has many appealing features. It contains the seeds of quark-lepton unification and involves only two gauge coupling constants $\left(g_{2 L}=g_{2 R}, g_{4 C}\right)$ which can be determined using low-energy data, most notably the ones from LEP. The boundary condition on Yukawa couplings necessary for the implementation of the see-saw mechanism holds starting from the GUT scale down to the intermediate scale. Another important consequence of this model is that all major sources of uncertainities like threshold and gravitational effects as well as those due to radiative corrections from higher scale $\left(\mu>M_{I}\right)$ do not affect the predicted values of $\sin ^{2} \theta_{W}$ [13] or $M_{I}$ [6] which have been proved through three theorems 6, 13].

There are claims in the literature [14] that the $G_{224}$ intermediate gauge symmetry with $M_{I}$ significantly different from $M_{G}$ is ruled out. Such an analysis utilises the right-handed triplet, $\left[\Delta_{R}(1,3, \overline{10})\right]$, under $G_{224}$, contained in the representation 126 of $S O(10)$ and its chiral conjugate, to break the intermediate gauge symmetry and to implement the see-saw mechanism. The large contributions of $\Delta_{R}$ and $\overline{\Delta_{R}}$ to the $\beta$ function disallow the possibility of $M_{I}$ being substantially lighter than the unification 
scale $\left(M_{G}\right)$ [14, 15]. Further, it is well known that the presence of $126+\overline{126}$ spoils perturbative grand unification above $\mu \geq 8 M_{G}$ in $S U S Y S O(10)$ and attempts of unification have been made through superstring-inspired particle spectrum [9].

Spontaneous breakdown of the intermediate gauge symmetry $G_{224}$ has also been carried out utilising the predicted representations [7] containing $16+\overline{16}$ of $S O(10)$. In this scenario the mass of the right handed majorana neutrino is generated either through non-renormalizable 5-dimensional opeartors or through purely renormalizable interaction by a novel mechanism [9]. The smaller contribution of the left and the right-handed doublets to the one-loop $\beta$ function in this case permits grand unification at the string- scale $M_{G} \simeq M_{\text {string }} \simeq 5 \times 10^{17} \mathrm{GeV}$, but having the intermediate scale substantially different; $M_{I} \simeq 10^{12}-10^{13} \mathrm{GeV}$. Such an intermediate symmetry needs the presence of lighter scalar superfields, $\sigma_{R}^{ \pm}(1, \pm 1,1), \chi_{3}\left(1, \frac{2}{3}, 3\right), \bar{\chi}_{3}\left(1,-\frac{2}{3}, \overline{3}\right)$ under the standard gauge group near the $\mathrm{TeV}$ scale and the $G_{224}$ submultiplet $\sigma_{C}(1,1,15)$ near the intermediate scale. While $\sigma_{R}^{ \pm}$and $\sigma_{C} \subset 45$ of $S O(10), \chi_{3}$ and $\bar{\chi}_{3}$ correspond to the would-be goldstone bosons $\subset 16+\overline{16}$, not absorbed in the process of intermediate breaking.

The contributions to the one loop $\beta$ functions in such a model at different energy scales turn out to be

$$
\begin{gathered}
M_{Z}<\mu<1 \mathrm{TeV} \quad b_{i}=\left(\begin{array}{c}
33 / 5 \\
1 \\
-3
\end{array}\right) \\
1 \mathrm{TeV}<\mu<M_{I}=10^{13} \mathrm{GeV} \quad b_{i}=\left(\begin{array}{c}
47 / 5 \\
1 \\
-2
\end{array}\right)
\end{gathered}
$$

where $i=Y, S U(2)_{L}, S U(3)_{c}$ and the extra light Higgs scalars, assumed to have masses $\mathcal{O}(1 \mathrm{TeV})$, contributes in eq. (2), and

$$
M_{I}<\mu<M_{G} \quad b_{i}=\left(\begin{array}{c}
7 \\
7 \\
2
\end{array}\right)
$$


where $i=S U(2)_{L}, S U(2)_{R}, S U(4)_{c}$ and the discrete $L-R$ symmetry is assumed for $\mu>M_{I}$.

Using $\sin ^{2} \theta_{W}=0.2315 \pm 0.0003, \alpha^{-1}\left(m_{Z}\right)=128.9 \pm 0.09$ and $\alpha_{3 c}^{-1}\left(m_{Z}\right)=0.119 \pm$ 0.004 [16] and the above $\beta$ functions in eqs (1-3) we find $\alpha_{2 R}\left(M_{I}\right)=0.039=\alpha_{2 L}\left(M_{I}\right)$, $\alpha_{4 c}\left(M_{I}\right)=0.059$ and $\alpha_{G U T}=0.074 \pm 0.004$..

Using the gluino mass $\left(m_{\tilde{g}}\right)$ at the EW scale as an input and the appropriate RG equations, we calculate the universal gaugino mass $M_{1 / 2}$ at the GUT scale. We then evolve downwards to calculate $U(1)_{Y}$ and $S U(2)_{L}$ gaugino masses $M_{1}$ and $M_{2}$ at the EW scale.

In this calculation we have taken into account the evolutions $M_{G} \rightarrow M_{I}, M_{I} \rightarrow$ $T e V$ scale and the $T e V$ scale $\rightarrow M_{Z}$ with appropriate $\beta$ functions given above. As shown in eqs (4.2)-(4.6), of [5] new $D$-term contributions, involving only one unknown parameter, introduce nonuniversality in the sfermion masses at $M_{I}$ for the first two generations and we focus our attention on the spectrum of this sector. In contrast the third generation and the Higgs scalars can acquire, in principle, both $D$ and $F$ term contributions to their masses at $M_{I}$ [5]. These sectors involving more unknown parameters are not included in our analysis. We shall, however, assume that $\tilde{b}_{L}$ and $\tilde{b}_{R}$ are approximately degenerate with $\tilde{d}_{L, R}$ and their masses at $M_{I}$ are also given by the formulae given below. We need this simplifying assumption to compare with the conventional phenomenology which assumes 5 degenerate squark flavours of both $L$ and $R$ type at a high scale. For $\mu \geq M_{I}$, the assumption of left-right discrete symmetry in $G_{224}$ constrains the masses of the left and the right chiral multiplets to be equal $\left(m_{L}=m_{R}=m_{0}\right)$, and, as compared to the formula given in [5], the number of parameters is reduced by one.

Using the values of the gauge-coupling constants given above we find for the masses of one generation of sfermions [5] at $\mu=M_{I}$ :

$$
\begin{aligned}
& m_{\tilde{q}}^{2}=m_{0}^{2}+0.74 D \\
& m_{\tilde{u}}^{2}=m_{0}^{2}+0.24 D \\
& m_{\tilde{e}}^{2}=m_{0}^{2}+1.24 D \\
& m_{\tilde{l}}^{2}=m_{0}^{2}-2.22 D
\end{aligned}
$$




$$
m_{\tilde{d}}^{2}=m_{0}^{2}-1.72 D
$$

where $\tilde{q}, \tilde{l}$ stand for doublets of squarks and sleptons, while $\tilde{d}, \tilde{u}$ and $\tilde{e}$ denote righthanded singlet squarks and sleptons.

In the above eqs. $D$ is an unknown parameter which may have either sign. The requirement of no tachyonic degrees of freedom at $M_{I}$, however, implies $D<$ $0.45 m_{0}^{2}$ (for $D>0$ ) from $m_{\tilde{l}}^{2}$ and $D>-0.81 m_{0}^{2}$ (for $D<0$ ) from $m_{\tilde{e}}^{2}$. In the subsequent phenomenological analyses, values of $D$ between these two extremes will be considered.

From eqs. (4) it follows that for $D>0$, the lepton doublet can be considerably lighter than the rest of the sfermions at $M_{I}$. Another notable feature is the suppression of $m_{\tilde{d}}^{2}$ compared to other squark masses. Suppression also affects $m_{\tilde{u}}^{2}$ although its magnitude is rather modest. On the other hand $D<0$, leaves open the possibility of a relatively light $\tilde{e}$ while the $\tilde{q}$ 's become the lightest squarks. We note that most of these features also hold at $M_{G}$ qualitatively if additional $D$-term contributions are taken into account in the one step breaking of $S U S Y S O(10)$ into the $S M$ gauge group (see eqs (4.9) - (4.13) of [5])

Using eqs (4) as boundary conditions and the gaugino masses, it is now straight forward to compute the sfermion masses for the first two generation at the EW scale. We obtain

$$
\begin{aligned}
m_{\tilde{u}_{L}}^{2} & =m_{0}^{2}+0.94 m_{\tilde{g}}^{2}+0.74 D+0.35 M_{Z}^{2} \cos 2 \beta \\
m_{\tilde{u}_{R}}^{2} & =m_{0}^{2}+0.91 m_{\tilde{g}}^{2}+0.24 D+0.15 M_{Z}^{2} \cos 2 \beta \\
m_{\tilde{d}_{L}}^{2} & =m_{0}^{2}+0.94 m_{\tilde{g}}^{2}+0.74 D-0.42 M_{Z}^{2} \cos 2 \beta \\
m_{\tilde{d}_{R}}^{2} & =m_{0}^{2}+0.90 m_{\tilde{g}}^{2}-1.72 D-0.07 M_{Z}^{2} \cos 2 \beta \\
m_{\tilde{\nu}}^{2} & =m_{0}^{2}+0.05 m_{\tilde{g}}^{2}-2.22 D+0.5 M_{Z}^{2} \cos 2 \beta \\
m_{\tilde{l}_{L}}^{2} & =m_{0}^{2}+0.05 m_{\tilde{g}}^{2}-2.22 D-0.27 M_{Z}^{2} \cos 2 \beta \\
m_{\tilde{l}_{R}}^{2} & =m_{0}^{2}+0.02 m_{\tilde{g}}^{2}+1.24 D-0.23 M_{Z}^{2} \cos 2 \beta
\end{aligned}
$$

The numbers quoted in these equations are obtained by using the central value of $\alpha_{G}$ given above.

Using $m_{0}$ (or equivalently the average left squark mass $m_{\tilde{q}}$ ), $m_{\tilde{g}}, \tan \beta$ and $D$ as the 
free parameters, one can study the entire sfermion spectrum. We, however, emphasise that certain broad mass patterns rather than very specific choices of the individual masses are responsible for interesting phenomenology. In the following we identify such patterns which yield $S U S Y$ signatures quite distinct from the conventional ones, leading to the possibility of revision of the current mass limits.

A) If $\mathbf{m}_{\mathbf{B}}^{\mathbf{2}}<<\mathbf{m}_{\tilde{\mathbf{g}}}^{\mathbf{2}}$, where $m_{B}$ generically represent the boundary values in eqs.(4) ,the nondegeneracy among the squarks at the EW scale is negligible in this case with all squarks having a common mass close to $m_{\tilde{g}}$. The squark sector is, therefore, very similar to the one in the conventional scenario. In contrast, the lepton doublet $\tilde{l}$ can still be much lighter compared to the conventional prediction for the same $m_{\tilde{g}}$ if $D>0$.

The striking phenomenological consequences of the light slepton scenario have already been emphasised in the literature [17, 18, 19]. Of particular interest is the case with $m_{\tilde{\nu}}<M_{\widetilde{\chi}_{1}^{ \pm}}$, where $\tilde{\chi}_{1}^{ \pm}$is the lighter chargino [18, 19]. As has been discussed already, in this case the $\tilde{\nu}$-s decay invisibly via the channel $\tilde{\nu} \rightarrow \nu \widetilde{N}_{1}$, where $\widetilde{N}_{1}$ is the lightest neutralino assumed to be the lightest sparticle (LSP), and the $\widetilde{\chi}_{1}^{ \pm}$decays via the 2-body decay mode $\tilde{\chi}_{1}^{ \pm} \rightarrow l^{ \pm} \tilde{\nu}$.

This mass pattern can also be accommodated in the conventional scenario. However, the available parameter space is rather narrow (see the region bounded by the two solid lines in Fig. 1, the other relevant $S U S Y$ parameters are chosen to be $\mu=-300.0 \mathrm{GeV}$ and $\tan \beta=2$ ). This perhaps is the reason for this scenario not being enthusiastically considered in most of the analyses. Once nonuniversal scalar masses at the intermediate scale are taken into account, the region expands significantly as is illustrated by the other lines in Fig. 1 which are obtained for different choices of $D$ (see the figure caption for further details). We have refrained from using choices numerically close to the extreme value $D=0.45 m_{0}{ }^{2}$ which extends the allowed region even more dramatically. In obtaining these regions we have taken into account the lower bounds on $m_{\tilde{e}_{R}}, m_{\tilde{\nu}}$ and $M_{\tilde{\chi}_{1}^{ \pm}}$derived from LEP [20]. Thus we find that relatively light $\tilde{\nu}$ 's look more probable within the basic framework of SUGRA once the theoretical uncertainities in the GUT scale/Planck scale physics are taken into account. 
One of the most important consequences of a light sneutrino is that the squarkgluino mass limits obtained at the Tevatron from the search in the jets $+\mathbb{Z}_{T}$ [21] channel are dependent on $m_{\tilde{\nu}}$ [19]. ur analyses reveal that this possibility is not confined to a tiny region of the parameter space. The effects of a light sneutrino should therefore be considered very seriously especially when sparticle mass limits are quoted for $m_{\tilde{q}} \approx m_{\tilde{g}}$.

Moreover, in view of the uncertainties in the magnitudes of $D$, it is not advisable to compute $m_{\tilde{\nu}}$ from any specific formula. Fortunately, the precise value of $m_{\tilde{\nu}}$ is unimportant for estimating its effects on the squark - gluino mass limits. As shown in [19 these limits are sensetive to $\Delta m=M_{\tilde{\chi}_{1}^{ \pm}}-m_{\tilde{\nu}}$. As long as $\Delta m$ is appreciable ( say $>20 \mathrm{GeV}$ ), one obtains conservative limits on $m_{\tilde{g}}$ and $m_{\tilde{q}}$. For small $\Delta m$, on the other hand, the limits become stronger. Thus the limits from the jets $+E_{T}$ channel play a complimentary role to those obtained from the dilepton [22] channel which become weaker for small $\Delta m[19]$.

B) Another interesting region of the parameter space is characterised by $m_{\tilde{q}}>>m_{\tilde{g}}$. In this case the sfermion masses at the EW scale are dominated by their boundary values at $M_{I}$. Thus sizable $D$-term contributions may indeed lead to significant mass hierarchies among the squarks. Such heavy squarks are of course beyond the kinematic limit of the Tevatron. In this scenario $S U S Y$ signals at Tevatron are expected to come primarily from gluino pair production with a cross section not very sensitive to $D$ (see Table I). Yet a significant mass split between the $\mathrm{L}$ and $\mathrm{R}$ squarks may indirectly influence the signal by drastically modifying the gluino branching ratio(BR)s compared to their values in the conventional scenario.

For $D>0$, the lightest among the squarks turns out to be of the $\tilde{d}_{R}$ type. For gluino masses accessible to the Tevatron the LSP is usually a pure Bino which couples favourably to these light $\tilde{d}_{R} \mathrm{~s}$. As a result $\operatorname{BR}\left(\tilde{g} \rightarrow \widetilde{N}_{1}+X\right)$ increases significantly since it is mediated by the light $\tilde{d}_{R}$ s. In contrast the conventional scenario corresponding to degenerate squarks ( $D=0)$ predicts dominantly cascade decays of the $\tilde{g}$. Thus the nonuniversality among the squark masses may lead to a $\not p_{T}$ spectrum much harder than the conventional one.

For $D<0$, on the other hand, $\tilde{q}_{L}$ s are likely to be the lightest species among the 
squarks which are favourably coupled to the $\widetilde{\chi}_{1}^{ \pm} \mathrm{s}$. Thus compared to the conventional scenario, the cascade decays of the gluino may become even more probable and direct decays of the gluino into the LSP may be reduced to an insignificant level provided the $\tilde{d}_{R}$ s are sufficiently heavy. This would soften the $\not p_{T}$ spectrum .

In Table I we present different BRs of $\tilde{g}$ decays to $\widetilde{\chi}_{1}^{ \pm}, \widetilde{N}_{1}$ and $\widetilde{N}_{2}$ (the second lightest neutralino) for some representative choices of $D$. We have chosen $m_{\tilde{g}}=250$ $\mathrm{GeV}$ and the mass of the heaviest squark ( either $\tilde{q}_{L}$ or $\tilde{d}_{R}$ ) to be $1 \mathrm{TeV}$. Through out this paper the c.m. energy is taken to be $2.0 \mathrm{TeV}$ corresponding to the proposed upgrade of the Tevatron. It is interesting to note that $\mathrm{BR}\left(\tilde{g} \rightarrow \widetilde{N}_{1}+X\right)$ varies between the two extremes of our choices: $D=0.30 m_{0}^{2}$ and $D=-0.56 m_{0}^{2}$ by a factor as large as 5 ! This strongly suggests that the effects of nonuniversality on the gluino mass limits for $m_{\tilde{q}}>>m_{\tilde{g}}$ should be reanalysed. Conservative limits are likely to be obtained in the jets $+E_{T}^{\prime}$ channel for $D<0$, since this case corresponds to the smallest production cross section (see Table I) and the softest $\not p_{T}$ spectrum (see below). In the dilepton channel on the other hand conservative limits are likely to follow for $D>0$..In either case the sensitivity of the current limits to phenomenological parameter $m_{\tilde{q_{L}}}-m_{\tilde{d}_{R}}$ is worth reexamination. In Fig 2 we present the $\not p_{T}$ spectrum for the above extreme values of $D$ as well as for $D=0$ ( the conventional scenario) using a parton level Monte Carlo. This illustrates the effects discussed above qualitatively . It is well known that a strong cut on $\not_{T}$ is crucial in seperating the $S U S Y$ signal from the background. It is therefore clear that nonconventional gluino branching ratios due to effects of GUT scale/Planck scale physics may affect the limits on $m_{\tilde{g}}$ for $m_{\tilde{q}}>>m_{\tilde{g}}$. Another important constraint on the $S U S Y$ signal is the requirement of three or four hard jets with $p_{T}>25 \mathrm{GeV}$ [21]. We have checked that this cut makes the difference between the three distributions in Fig. 2 even more prominent. However, we do not present the details here since putting the experimental cuts on the parton jets may not be completely realistic.

C) The third region of the parameter space which we find phenomenologically interesting corresponds to squarks moderately heavier than gluinos. However, both $m_{\tilde{q}}$ and $m_{\tilde{g}}$ are within the kinematic reach of the Tevatron. In this case the mass hierarchy among the squarks may not be as large as in case B), yet $m_{\tilde{q}_{L}}-m_{\tilde{d}_{R}}$ can be 
numerically significant (see Table II). In Fig.1 the region of the $m_{\tilde{q}}-m_{\tilde{g}}$ plane above the upper dot dashed line corresponds to $m_{\tilde{q}}-m_{\tilde{d}_{R}}>25 \mathrm{GeV}$ for $D>0.16 m_{0}^{2}$, while that above the upper dashed line correspond to $m_{\tilde{d}_{R}}-m_{\tilde{q}}>25 \mathrm{GeV}$ for $D<-0.16 m_{0}^{2}$. Even such modest mass splittings turn out to be sufficient to make the total squark - gluino production cross section $\sigma_{\tilde{q} \tilde{g}}$ appreciably different from the conventional predictions corresponding to $D=0$. The difference is basically driven by the production of gluinos in association with a relatively light squark. This is illustrated in Table II where we present $\sigma_{\tilde{q} \tilde{g}}$ for different choices of $D$. We have chosen $m_{\tilde{g}}=230 \mathrm{GeV}$ and the mass of heaviest squark $\left(\tilde{q}_{L}\right.$ for $D>0$ and $\tilde{d}_{R}$ for $\left.D<0\right)=300 \mathrm{GeV}$. Since there are five squark flavours of the $\tilde{q}_{L}$ type, relatively light $\tilde{q}_{L} \mathrm{~s}$ affect the cross section quite drastically (see Table II ).

Although in this case the branching ratios of squark/gluino decays are rather similar to the conventional ones, the size of the SUSY signals can be quite different due to the above modification in the cross sections. It is to be noted that for an integrated luminosity of $1 \mathrm{fb}^{-1}$, which can be accumulated after the luminosity upgrade of the Tevatron, the total number of $\tilde{q}-\tilde{g}$ events ( Table 2 ) are strongly affected by modest nonuniversalities among the squark masses, which are quite probable in view of the uncertainties in Planck scale/GUT scale physics.

Although our numerical analyses are restricted to scenarios relevant for SUSY searches at Tevatron, we have checked that nonuniversality of sfermion masses may lead to similar significant effects at LHC. For example, the heavy squarks discussed in case B above, are accessible to LHC. Therefore nonuniversality in their masses may affect the SUSY signals not only through the gluino branching ratios but also through the total squark-gluino production cross section. For extreme positive values of $D$, mass hierarchies such as $m_{\tilde{q_{L}}}>m_{\tilde{g}}>m_{\tilde{d}_{R}}$ are also allowed. In this case $\operatorname{Br}\left(\tilde{g} \rightarrow \widetilde{N}_{1}+X\right)$ becomes $100 \%$ making the resulting $\not_{T}$ spectrum very hard.

In conclusion we reiterate that possible variations in GUT/Planck scale physics may significantly alter the SUSY signals and the existing mass limites from the Tevatron, expected in the conventional scenario. We have identified three mass patterns which might lead to signals quite distinct from the ones predicted conventionally. All these patterns may arise naturally in variations of $N=1 S U G R A$ motivated models 
with additional nonuniversal $D$ terms at a high scale $\left(M_{I}\right.$ or $\left.M_{G}\right)$. For $m_{\tilde{q}} \simeq m_{\tilde{g}}$, the existing mass limits on the sparticles should be reexamined keeping in view the possibility of a relatively light sneutrino $\left(m_{\tilde{\nu}}<M_{\tilde{\chi}_{1}^{ \pm}}\right)$. For $m_{\tilde{q}}>>m_{\tilde{g}}$, nonuniversality in the suark masses may modify the signal by changing the gluino branching ratios anticipated in the scenario with universal soft breaking masses for the sfermions. This may lead to revisions of the gluino mass limits. For squarks moderately heavier than the gluinos but both having masses within the kinematic limit of Tevatron, even modest nonuniversality among the squark masses may affect the signal and the existing squark-gluino mass limits by modifying the total squark-gluino cross sections.

Although for the purpose of illustration we have restricted our numerical results mostly to $S O(10)$ SUSY GUT with intermediate mass scales, many of our conclusions are also valid even if the GUT directly breaks down to the MSSM.

Acknowledgement: The work of AD was supported by grants from the Department of Atomic Energy and the Department of Science and Technology, Govt. of India. The work of Aseshkrishna Datta was supported by the Council of Scientific and Industrial Research, Govt. of India. MKP thanks Professors G.Senjanovic,A.Yu.Smirnov, S.Randjbar-Daemi, High Energy Group and the International Centre for Theoretical Physics, Trieste, Italy for hospitality as ICTP Associate.

\section{References}

[1] R. Arnowitt, A. H. Chamseddine and P. Nath, Phys. Rev. Lett. 49 (1982) 970; R. Barbieri, S. Ferrara and C. A. Savoy, Phys. Lett. B119 (1982) 343; L. J. Hall, J. Lykken and S. Weinberg, Phys. Rev. D27 (1983) 2359.

[2] A large number of papers have recently been published on this subject a partial list is: M. Olechowski and S. Pokorski, Phys. Lett. B344 (1995) 201 ; T. Kobayashi, D. Suematsu, K. Yamada and Y. Yamagishi, Phys. Lett. B348 (1995) 402; See also refs 3,4 and 5 . 
[3] P. Moxhay and K. Yamamoto, Nucl. Phys. 256B, (1985) 130 ; B. Gato, ibid 278B (1986) 189; N. Polonsky and A. Pomarol, Phys. Rev. D51 (1995) 6532.

[4] M. Drees, Phys. Lett. B181 (1986) 279.

[5] Y. Kawamura, H. Murayama and M. Yamaguchi, Phys. Rev. D51 (1995) 1337.

[6] M.K.Parida, ICTP Preprint IC/96/33, hep-ph/9710246, to appear in Phys.Rev. D.

[7] S.Chaudhuri, S.W.Chung, G.Hockney and J.Lykken, hep-ph/9501361.

[8] M.Gell-Mann, P.Ramond and R.Slansky in Supergravity, eds. P.van Niewenhuizen and D.Z.Freedman (North Holland,1979); T. Yanagida, in Proceedings of the Workshop on Unified Theory and Baryon Number of the Universe, eds. O.Sawada and A.Sugamoto(KEK, 1979): R.N.Mohapatra and G.Senjanovic, Phys.Rev.Lett. 44, (1980) 912 ; Phys.Rev. D23 (1981) 165.

[9] Dae Gyu Lee and R.N.Mohapatra, Phys.Rev. D52 (1995) 4125.

[10] A.Yu. Smirnov, Nucl.Phys. B466 (1996) 25;M.K.Parida, S.Dey and B.Purkayastha(In preparation).

[11] S.P.Mikheyev and A.Yu.Smirnov, Yad.Fiz. 42 (1975) 1441 ; L.Wolfenstein Phys.Rev. D17 (1979) 2369.

[12] J. C. Pati and A. Salam, Phys. Rev. Lett. 31 (1973) 661.

[13] M.K.Parida and P.K.Patra, Phys.Rev.Lett. 68 , (1992) 754 ; Phys.Rev.Lett. 66 (1991) 858 .

[14] M.Bando, J.Sato and T.Takahasi, Phys.Rev. D52 (1995) 3074.

[15] K.Benakli and G.Senjanovic, Phys.Rev. D54 (1996) 5734 .

[16] Particle Data Group Phys. Rev. D54 (1996) 1:G.Altarelli, hep-ph/9710434.

[17] H. Baer, C. Kao and X. Tata, Phys. Rev. D48 (1993) R2978 ; R. M. Barnett, J. Gunion and H. Haber, Phys. Lett. B315 (1993) 349. 
[18] A. Datta, B. Mukhopadhyaya, and M. Guchhait, Mod. Phys. Lett., 10, (1995) 1011; A. Datta, M. Drees, and M. Guchhait, Z. Phys. C69 (1996) 347; S. Chakraborty, A. Datta, and M. Guchait, Z. Phys. C68 (1995) 325; A. Datta, Aseshkrishna Datta, S. Raychaudhuri, Phys. Lett. B349 (1995) 113; A. Datta, Aseshkrishna Datta, S. Raychaudhuri, hep-ph/9605432, to appear in Z. Phys. C.

[19] A. Datta, M. Guchait, N. Parua, Phys. Lett. B395 (1997) 54.

[20] ALEPH Collaboration, D. Buskulic et al, CERN-PPE/97-056 ; The OPAL Collaboration, K. Ackerstaff et al, Phys. Lett. B396, (1996) 301 ; The OPAL Collaboration, K. Ackerstaff et al, Phys. Lett. B389 (1996) 616.

[21] CDF Collaboration, F. Abe et al, Phys. Rev. Lett., 69 (1992) 3439; D0 Collaboration, S. Abachi et al, Phys. Rev. Lett. 75 (1995) 619.

[22] CDF Collaboration, F.Abe et al, Phys.Rev.Lett., 76 (1996) 2006. 


\section{Table Captions :}

Table-I : Branching Ratios for gluino decays (see text) and gluino pair production cross section for $m_{\tilde{q}}>>m_{\tilde{g}}$ in a $S O(10) S U S Y$ GUT with an intermediate scale for different values of $D$.

Table-II : The total squark-gluino production cross section and the total number of squark-gluino events for an integrated luminosity of $1 \mathrm{fb}^{-1}$ with squarks moderately heavier than the gluino in a $S O(10) S U S Y$ GUT with an intermediate scale for different values of $D$.

\section{Figure Captions :}

Fig. 1 The regions in the $m_{\tilde{q}}$ (average left squark mass) $-m_{\tilde{g}}$ plane where (a) light $\tilde{\nu}$ 's $\left(M_{\tilde{\chi}_{1}^{ \pm}}>m_{\tilde{\nu}}\right)$ or (b) significant mass difference between the L and R squarks may arise in different SUSY GUTs. The region bounded by the solid curves satisfies criterion (a) in the conventional scenario. The long dashed line represents the upper edge of the allowed region (type a) in a grand desert type $S O(10)$ SUSY GUT with $D=0.16 m_{0}{ }^{2}$. The dotted line (lower dot-dashed line) corresponds to the same upper edge in a $S O(10) S U S Y$ GUTs with an intermediate scale for $D=0\left(D=0.16 m_{0}{ }^{2}\right)$. In the region above the upper dot-dashed line (short dashed line) $m_{\tilde{q}_{L}}-m_{\tilde{d}_{R}}>25$ $\mathrm{GeV}\left(m_{\tilde{d}_{R}}-m_{\tilde{q}_{L}}>25 \mathrm{GeV}\right)$ in an intermediate scale $S O(10) S U S Y$ GUT with $D=0.16 m_{o}{ }^{2}\left(D=-0.16 m_{o}{ }^{2}\right)$. Through out this paper we take $\mu=-300$ and $\tan \beta=2$.

Fig. 2 The $\not p_{T}$ spectrum for gluino decays in $S O(10) S U S Y$ GUT with an intermediate scale. We have taken $m_{\tilde{g}}=250 \mathrm{GeV}$ and the heaviest squark mass equal to 1 $\mathrm{TeV}$. The solid, dashed and dot-dashed curves correspond to $D=0, D=0.30 m_{0}{ }^{2}$ and $D=-0.56 m_{o}^{2}$ respectively. 
Table I

\begin{tabular}{|c|c|c|c|c|c|c|c|}
\hline$\frac{D}{m_{o}{ }^{2}}$ & $m_{\tilde{q}_{L}}$ & $m_{\tilde{u}_{R}}$ & $m_{\tilde{d}_{R}}$ & $\operatorname{Br}\left(\widetilde{\chi}_{1}^{ \pm}\right)$ & $\operatorname{Br}\left(\widetilde{N}_{1}\right)$ & $\operatorname{Br}\left(\widetilde{N}_{2}\right)$ & $\sigma_{\text {total }}$ in $\mathrm{pb}$ \\
\hline 0.30 & 1000 & 930 & 583 & 33.6 & 45.3 & 21.0 & 1.64 \\
\hline 0.16 & 1000 & 965 & 817 & 45.5 & 26.4 & 28.1 & 1.67 \\
\hline 0 & 1000 & 1000 & 1000 & 49.3 & 20.3 & 30.4 & 1.70 \\
\hline-0.16 & 842 & 876 & 1000 & 51.6 & 16.6 & 31.8 & 1.55 \\
\hline-0.56 & 542 & 661 & 1000 & 56.1 & 9.4 & 34.5 & 1.16 \\
\hline
\end{tabular}

Table II

\begin{tabular}{|c|c|c|c|c|c|}
\hline$\frac{D}{m_{o}{ }^{2}}$ & $m_{\tilde{q}_{L}}$ & $m_{\tilde{u}_{R}}$ & $m_{\tilde{d}_{R}}$ & $\sigma_{\text {total }}$ in pb & No. of events \\
\hline 0.30 & 300 & 288 & 246 & 3.57 & 3570 \\
\hline 0.16 & 300 & 293 & 274 & 3.29 & 3290 \\
\hline 0 & 300 & 300 & 300 & 2.99 & 2990 \\
\hline-0.25 & 269 & 274 & 300 & 4.24 & 4240 \\
\hline-0.56 & 242 & 253 & 300 & 6.00 & 6000 \\
\hline
\end{tabular}




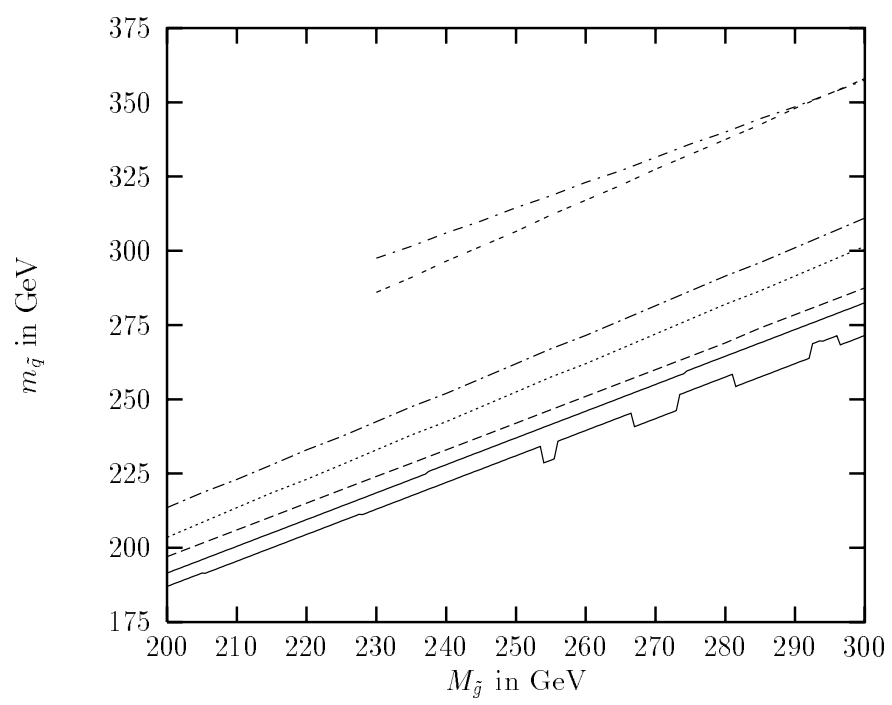

Figure 1:

1 


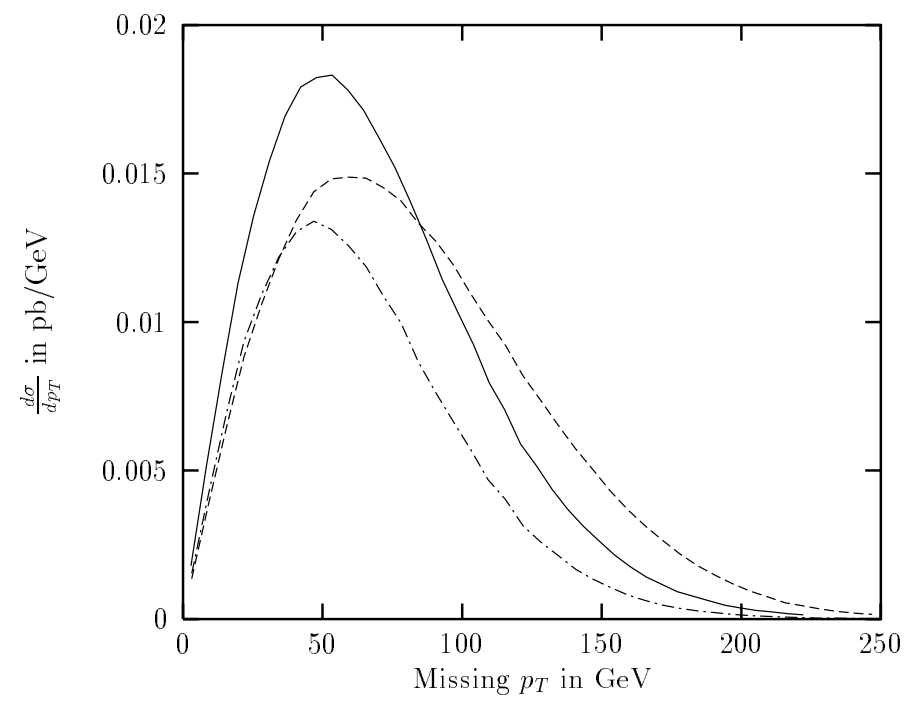

Figure 2: 